\title{
SI: Hygroscopicity of Microplastic and Mixed Microplastic Aqueous Ammonium Sulfate Systems
}

\author{
July 30, 2021 \\ Alison Bain and Thomas C. Preston* \\ Department of Atmospheric and Oceanic Sciences \\ and Department of Chemistry, \\ McGill University, 805 Sherbrooke Street West, Montreal, QC, Canada H3A 0B9 \\ submitted to Environmental Science and Technology \\ 10 Figures and 6 manuscript pages
}

* Thomas C. Preston

e-mail: thomas.preston@mcgill.ca 


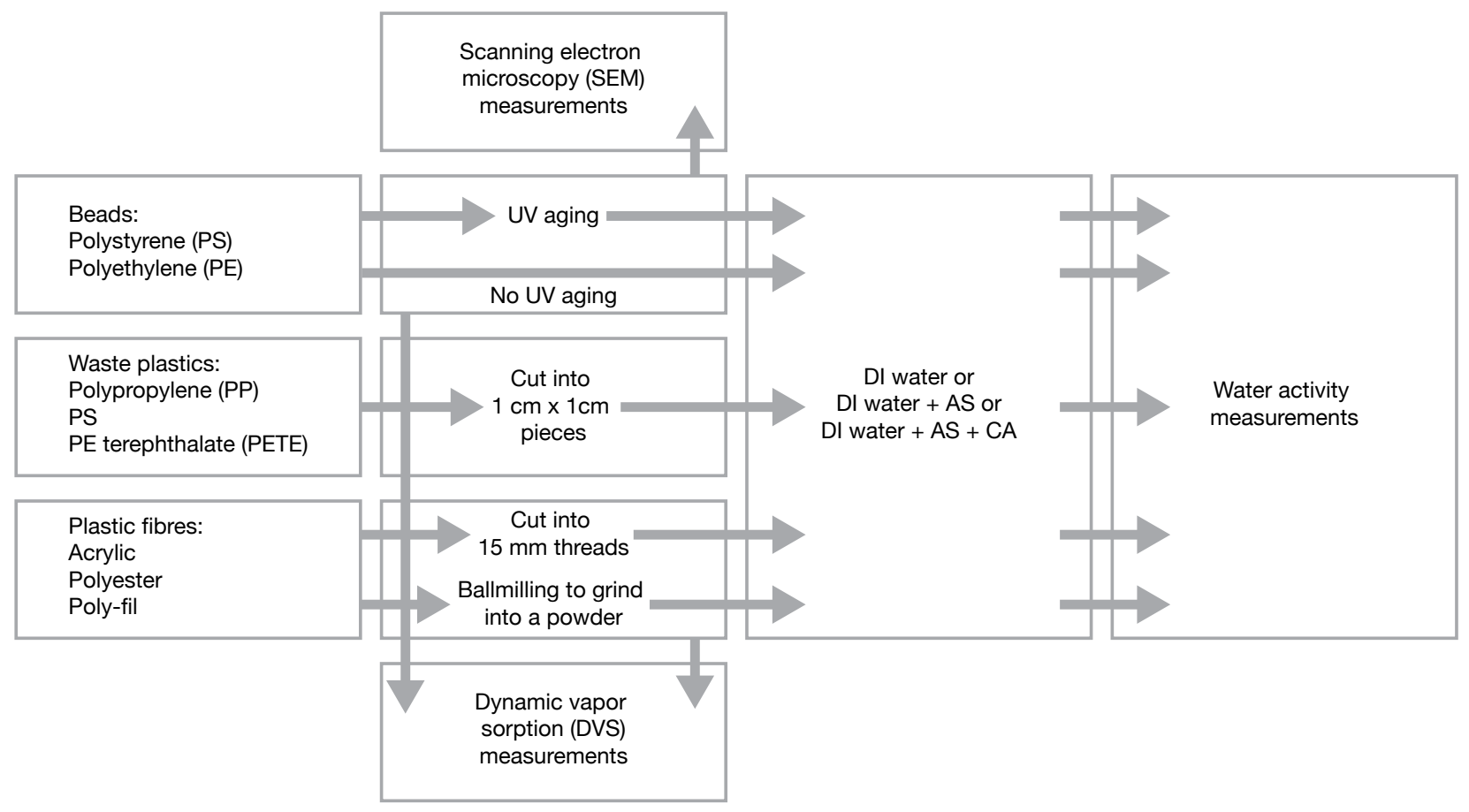

Figure S1: Schematic of experimental steps. 


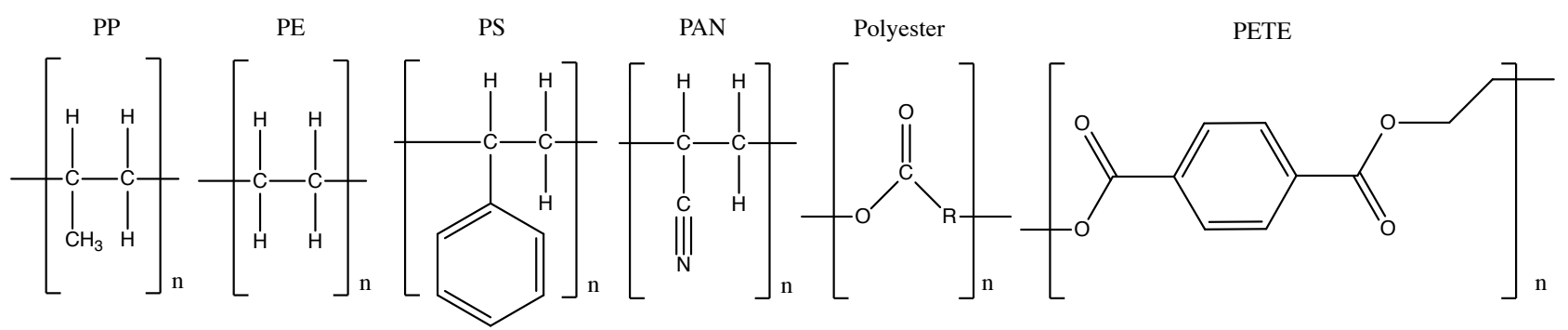

Figure S2: Chemical structures for polymers investigated. Polypropylene (PP), polyethylene (PE), polystyrene (PS), polyacrylonitrile (PAN) which is the major monomer in acrylic copolymers, Polyester and polyethylene terephthalate (PETE)
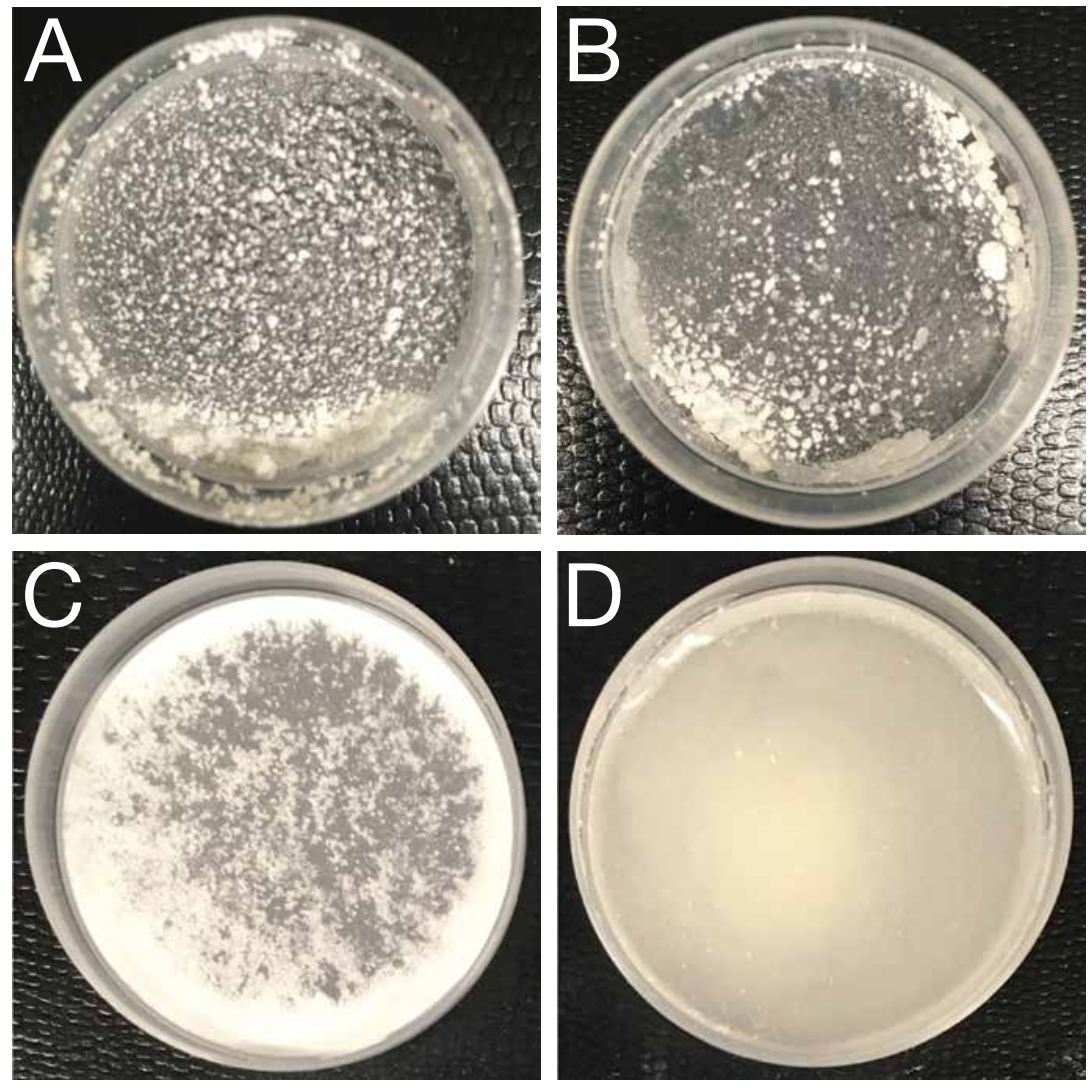

Figure S3: Samples containing A) pristine PE beads, B) pristine PS beads, C) aged PE beads and D) aged PS beads. Plastic mass fraction is approximately 0.01 in each. 


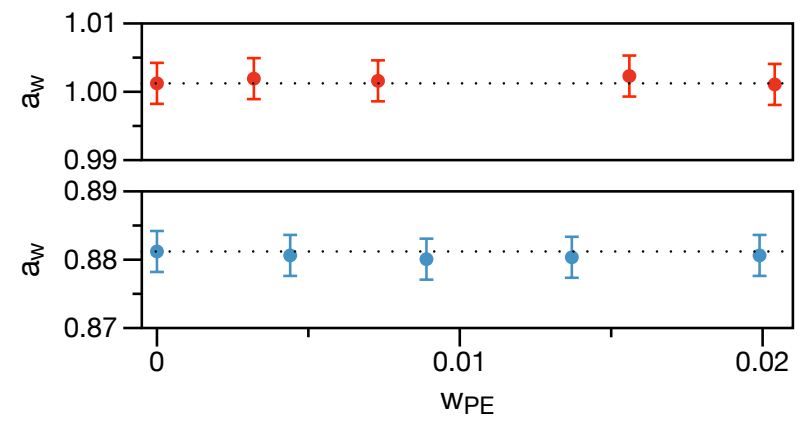

Figure S4: Average water activity for DI water (top) and aqueous AS (bottom) as a function of pristine PE bead mass fraction. Grey dotted lines indicate the average $a_{\mathrm{w}}$ measured before the addition of plastic.

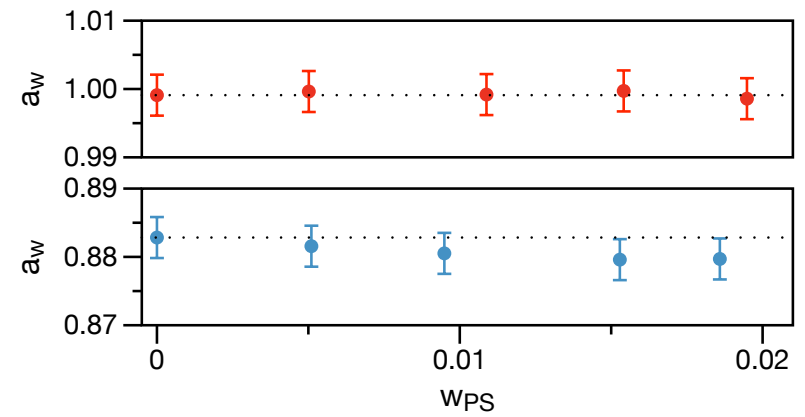

Figure S5: Average water activity for DI water (top) and aqueous AS (bottom) as a function of pristine PS bead mass fraction. Grey dotted lines indicate the average $a_{\mathrm{w}}$ measured before the addition of plastic.

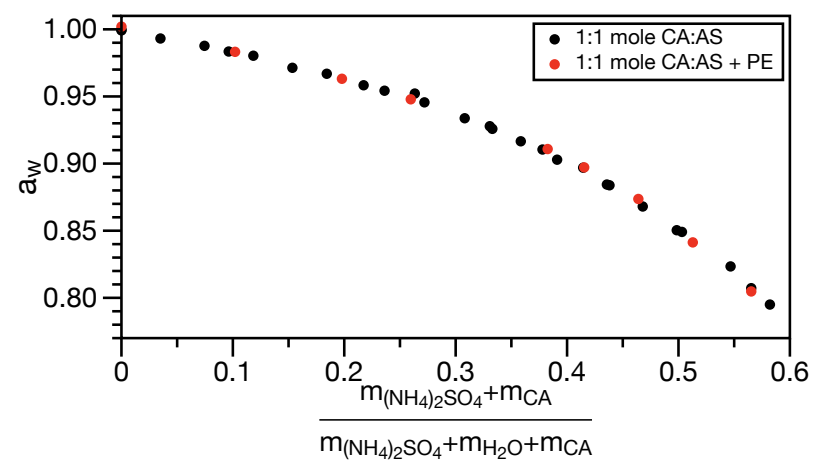

Figure S6: Water activity of aqueous 1:1 mole CA:AS (citric acid to ammonium sulfate) with PE beads. The total mass fraction of solute, neglecting the mass of the beads is shown on the $\mathrm{x}$-axis. 


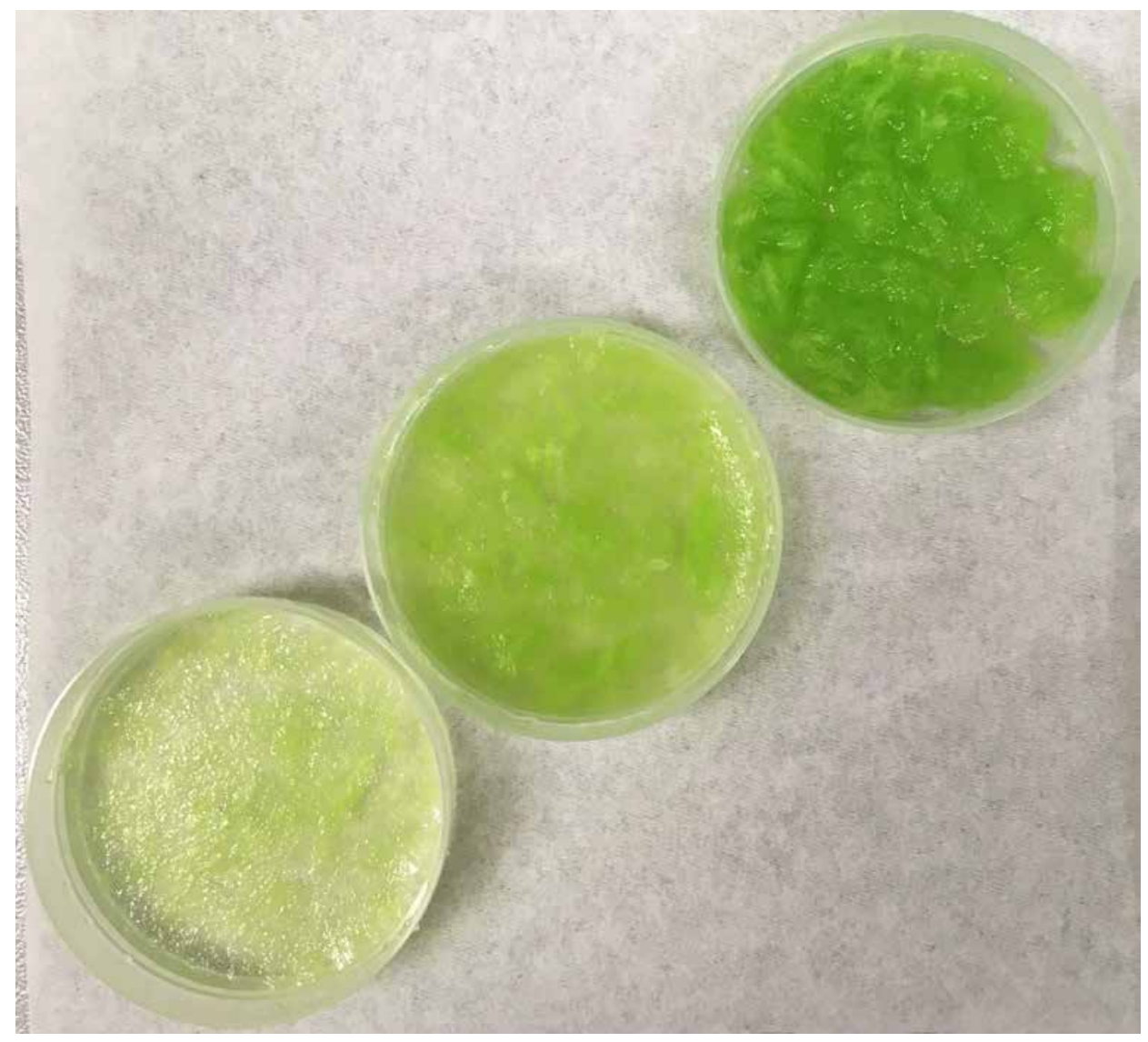

Figure S7: Example photo of cut acrylic yarn fibers mixed with deionized water at a range of acrylic mass fractions for bulk water activity measurements.

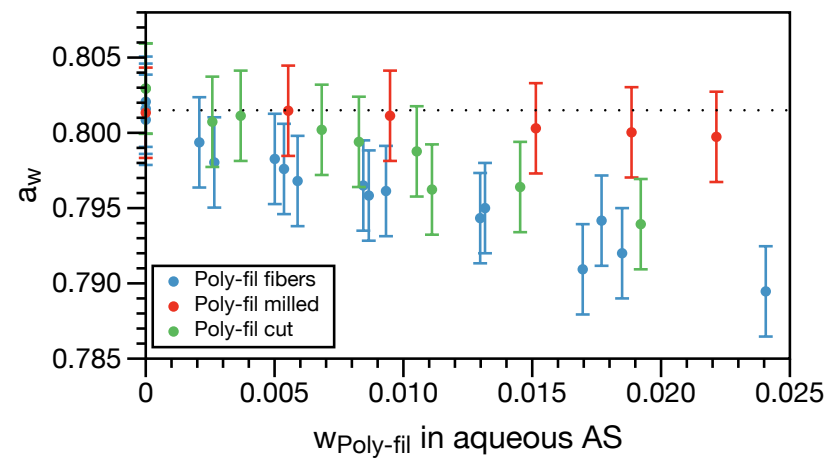

Figure S8: Water activity of near saturated AS solution with the addition of whole, cut and milled Poly-fil fibers. 


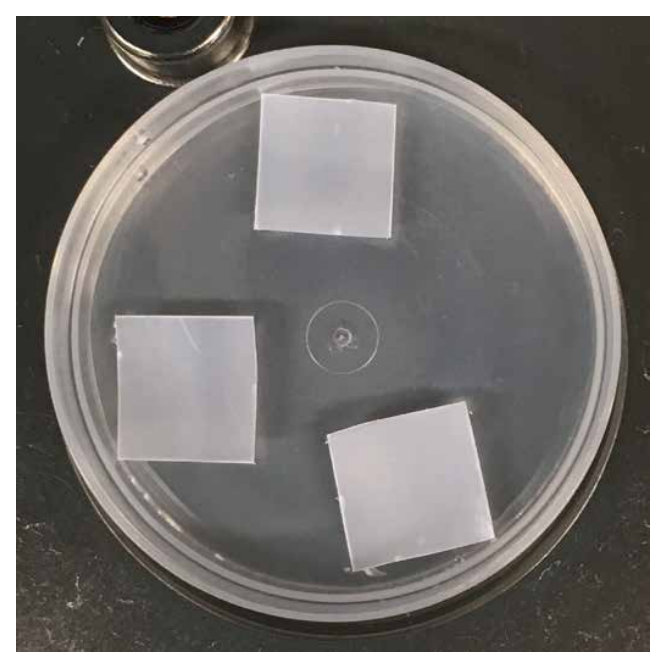

Figure S9: Example photo of polypropylene $1 \mathrm{~cm}^{2}$ squares in a sample cup with water for the effect of surface coverage bulk water activity measurements.
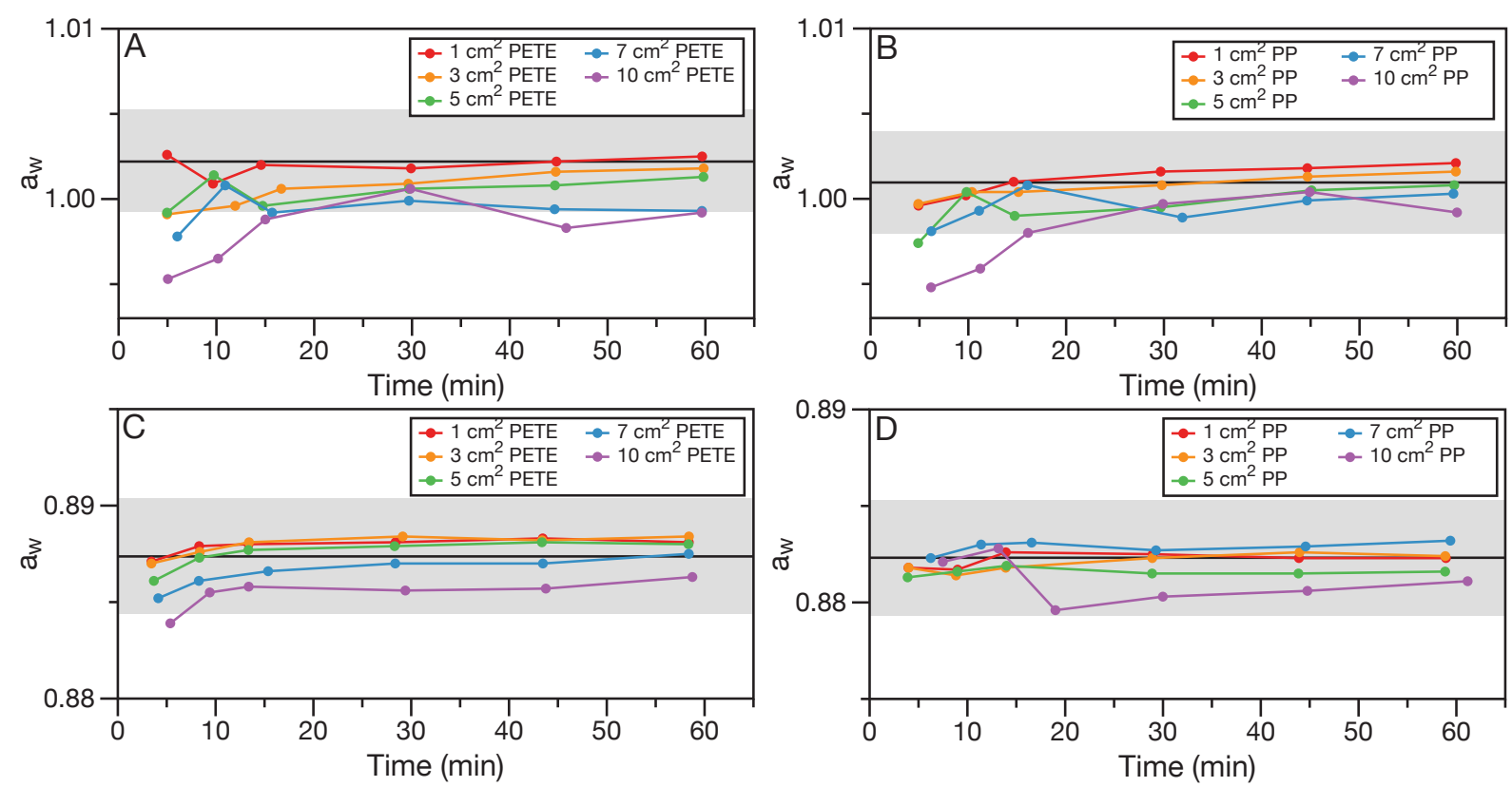

Figure S10: Measured water activity as a function of time and $1 \mathrm{~cm}^{2}$ waste plastic pieces. Solid black line indicates the average $a_{\mathrm{w}}$ measured before the addition of plastic and shaded grey region shows the \pm 0.003 instrument read error. A) PETE and deionized water, B) PP and deionized water, C) PETE and aqueous ammonium sulfate, D) PP and aqueous ammonium sulfate. 

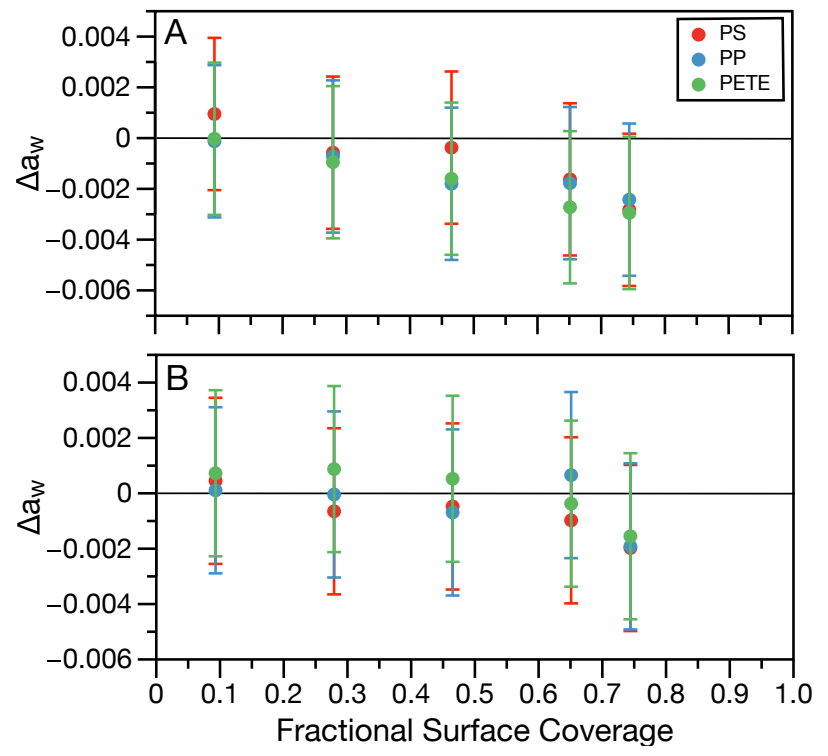

Figure S11: Change in measured water activity between aqueous sample and sample with waste plastic covering a fraction of the surface of A) deionized water, B) aqueous AS. $\Delta a_{\mathrm{w}}$ is calculated by subtracting the average water activity for samples containing plastics from the samples without plastics. The average water activity for samples containing plastics was calculated using only the final four measurements from each run ( $t>13$ minutes) to ensure equilibrium was reached. Error bars represent the instrument read error. Standard deviations in the measurements are smaller than the instrument read error. $\Delta a_{\mathrm{w}}>0$ indicates the addition of plastic increased the measured water activity and $\Delta a_{\mathrm{w}}<0$ indicates the addition of plastic decreased the measured water activity. 